\title{
First evidence of a collision between two unrelated open clusters in the Milky Way
}

\author{
Andrés E. Piatti ${ }^{1,2 \star}$ and Khyati Malhan ${ }^{3,4}$ \\ ${ }^{1}$ Instituto Interdisciplinario de Ciencias Básicas (ICB), CONICET-UNCUYO, Padre J. Contreras 1300, M5502JMA, Mendoza, Argentina \\ ${ }^{2}$ Consejo Nacional de Investigaciones Cientificas y Técnicas, Godoy Cruz 2290, C1425FQB, Buenos Aires, Argentina \\ ${ }^{3}$ The Oskar Klein Centre for Cosmoparticle Physics, Department of Physics, Stockholm University, AlbaNova, 10691 Stockholm, Sweden \\ ${ }^{4}$ Max-Planck-Institut für Astronomie, Königstuhl 17, D-69117, Heidelberg, Germany
}

Accepted XXX. Received YYY; in original form ZZZ

\begin{abstract}
We report the first evidence of an on-going collision between two star clusters in our Galaxy, namely IC 4665 and Collinder 350. These are open clusters located at a distance of $\sim 330 \mathrm{pc}$ from the Sun and $\sim 100 \mathrm{pc}$ above the Galactic plane, and they both have prograde motions with only a small difference in their velocities (Collinder 350 moves $\sim 5 \mathrm{~km} \mathrm{~s}^{-1}$ faster than IC 4665); as inferred from ESA/Gaia based catalogue. Interestingly, the two clusters are physically separated by only $\sim 36 \mathrm{pc}$ in space; a distance that is smaller than the sum of their respective radii. Furthermore, the clusters exhibit signatures of elongated stellar density distributions, and we also detect an onset of an inter-cluster stellar bridge. Moreover, the orbit analysis suggests that the younger cluster IC 4665 (age=53 Myr) must have formed at a distance $>500 \mathrm{pc}$ away from Collinder 350 (age=617 Myr). These findings together imply that the two clusters do not represent merging of two objects in a binary system, rather, what we are witnessing is an actual collision between two independently formed star clusters. This collision phenomenon provides a unique opportunity to explore new aspects of formation and evolution theory of star clusters.
\end{abstract}

Key words: Methods: observational - Methods: numerical - Galaxy: open clusters and associations: individual: IC 4665, Collinder 350

\section{INTRODUCTION}

The Milky Way is populated by thousands of open clusters, and most of them observed as independent objects. Here, we use the classification of open cluster given by Bica et al. (2019, see their Table 1), who differentiated open clusters from associations, embedded clusters, cluster remnants, etc (see, e.g. Feigelson et al. 2011; Kounkel et al. 2018; Townsley et al. 2018; Cantat-Gaudin et al. 2019; Kerr et al. 2021). Binary clusters are pairs of clusters that posses small physical separation.

de la Fuente Marcos \& de la Fuente Marcos (2010) estimated that nearly $10 \%$ of the total population of open clusters could in fact be binary systems. However, the number of physical pairs identified by de La Fuente Marcos \& de La Fuente Marcos (2009) increases up to only 34 candidates, with a best candidate sample of only 19 bonafide pairs. Interestingly, de La Fuente Marcos \& de La Fuente Marcos (2009) arrived at this result based on the distinction between couple and binary clusters, after considering

* E-mail: andres.piatti@unc.edu.ar that clusters may form as pairs, triplets or higher multiplicity star clusters. Conrad et al. (2017) used radial velocities from the RAdial Velocity Experiment (RAVE Steinmetz et al. 2006) catalogue, and also other catalogues, to identify only 19 pairs, and among these pairs only 14 pairs possessed physical separations smaller than 100 pc. Soubiran et al. (2018) employed the Gaia DR2 database (Gaia Collaboration et al. 2016, 2018a) to analyse 861 open clusters in the local $6 \mathrm{D}$ phase space volume and identified only 5 physical pairs. They concluded that all the previously identified binary clusters were chance alignments. It is important to find bonafide pairs of open clusters as they provide ideal laboratories to constrain the still poorly understood formation and evolution theory of star clusters.

The above overview reveals that binary open clusters are not very common in the Milky Way. Indeed, de la Fuente Marcos \& de la Fuente Marcos (2010) showed from simulations that long-lasting binary open clusters are not very stable systems. They found that cluster pairs that initially form together quickly undergo merging. They reported that the physical separation between the pairs peaks at $25-30 \mathrm{pc}$. With the aim of examining the above results, here we used 


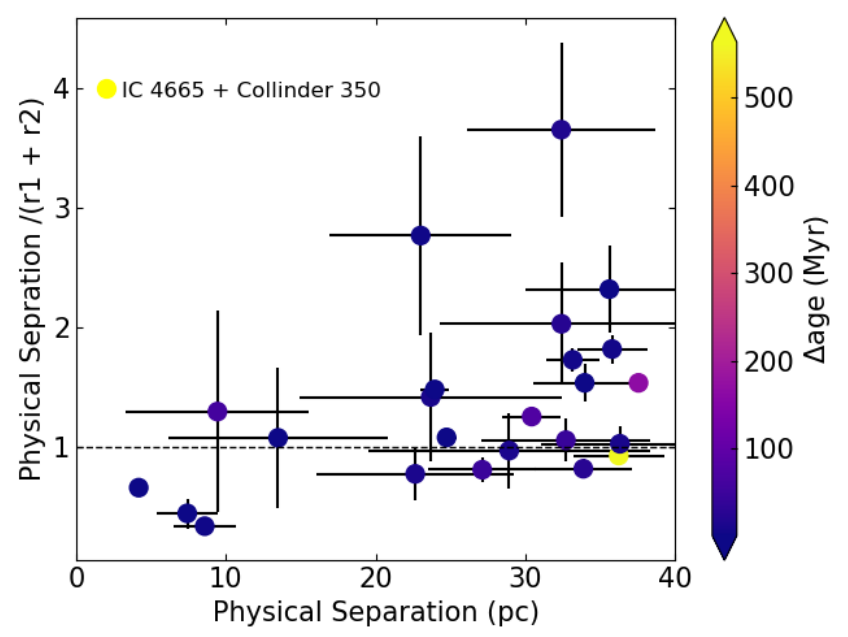

Figure 1. Ratio of the physical separation to the sum of the cluster adopted radii versus $3 \mathrm{D}$ cluster separation. Cluster pairs below the dotted line are thought to be experiencing merging.

the recently updated open cluster catalogue compiled by Dias et al. (2021). This catalogue includes homogeneous astrophysical properties derived for 1743 open clusters from Gaia DR2 data sets (Gaia Collaboration et al. 2016, 2018a). We performed a search for cluster pairs with physical separation smaller than $40 \mathrm{pc}$ and compared those distances with the sum of their adopted radii $\left(r_{1}+r_{2}\right)$. To calculate the corresponding uncertainties we used a Monte Carlo sampling of the involved parameters and their uncertainties.

Figure 1 shows that out of 1743 open clusters, the number of close pairs with separations smaller than $40 \mathrm{pc}$ is relatively small (although we note that at this stage we did not consider the relative velocities between the two clusters). All detected pairs, but one, are composed of open clusters that possess similar ages ( $\Delta$ age $\lesssim 100 \mathrm{Myr}$ ), implying that these binary pairs were formed at the same time (likely at the same location in our Galaxy). This inference is in very good agreement with our previous knowledge of the age difference of physical binary clusters (de La Fuente Marcos \& de La Fuente Marcos 2009; Conrad et al. 2017; Soubiran et al. 2018). We additionally note that those binary clusters with ratios of the physical separation to the sum of the tidal radii smaller than the unity (dotted line in Fig. 1) are experiencing merging (de la Fuente Marcos \& de la Fuente Marcos 2010). Interestingly, Figure 1 shows that there exists one cluster pair, located below the dotted line, with an age difference $>500 \mathrm{Myr}$. This peculiar cluster pair corresponds to the system comprising of two open clusters - IC 4665 and Collinder 350 .

In this work, our aim is to report the discovery of this first observed collision between these two open clusters IC 4665 and Collinder 350. In Section 2, we describe the observation evidence that supports the cluster collision, which Section 3 deals with numerical simulations of the clusters' orbits. Section 4 presents the final conclusions and discussion of this work.

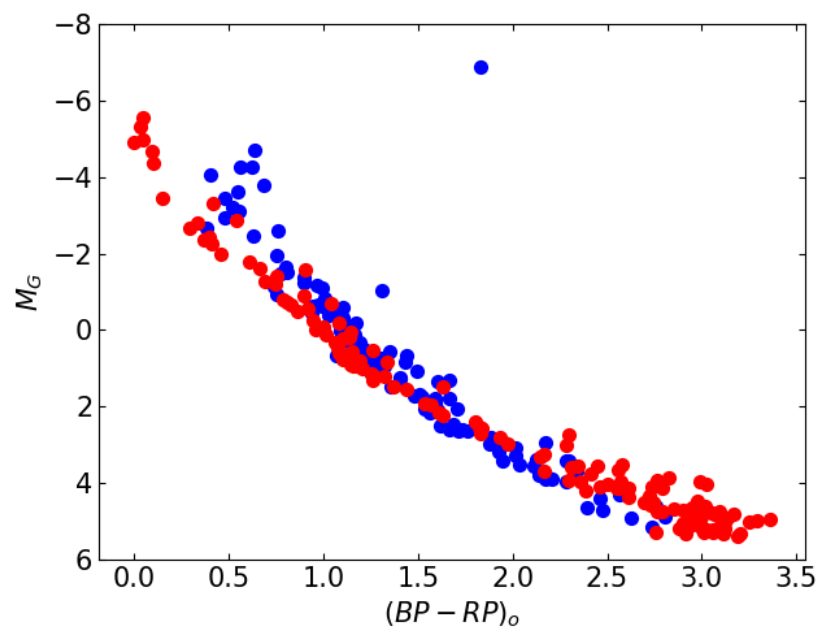

Figure 2. Colour-magnitude diagram for cluster members in Collinder 350 and IC 4665, represented with filled blue and red circles, respectively.

\section{THE OBSERVED CLUSTER COLLISION}

IC 4665 has an age of $53 \mathrm{Myr}$, and its phasespace position in the Galaxy in heliocentric coordinates is $\left(\mathrm{ra}\right.$, dec. $\left._{\mathrm{f}} \mathrm{d}_{\odot}, \mu_{\alpha}^{*}, \mu_{\delta}, \mathrm{v}_{\mathrm{r}}\right)=\left(266.5541^{\circ}, 5.5800^{\circ}\right.$, $0.329 \pm 0.015 \mathrm{kpc}, \quad-0.896 \pm 0.317 \mathrm{mas} \mathrm{yr}^{-1}, \quad-8.504 \pm$ $0.328 \mathrm{mas} \mathrm{yr}^{-1}, \quad-14.142 \pm 4.351 \mathrm{~km} \mathrm{~s}^{-1}$ ). Collinder 350 has an age of $617 \mathrm{Myr}$, and its phase-space position is $\left(\mathrm{ra}, \mathrm{dec}_{\mathrm{g}}, \mathrm{d}_{\odot}, \mu_{\alpha}^{*}, \mu_{\delta}, \mathrm{v}_{\mathrm{r}}\right)=\left(266.9397^{\circ}, 1.5473^{\circ}, 0.356 \pm\right.$ $0.010 \mathrm{kpc},-4.928 \pm 0.412 \mathrm{mas} \mathrm{yr}^{-1},-0.020 \pm 0.274 \mathrm{mas} \mathrm{yr}^{-1}$, $-14.665 \pm 1.309 \mathrm{~km} \mathrm{~s}^{-1}$ ) (Dias et al. 2021). As one can note, although IC 4665 and Collinder 350 are separated in the sky by $\sim 4^{\circ}$, their volumes seem to be superimposed. This can be inferred when considering their dimensions as estimated by Dias et al. (2021), i.e., their radii estimated as the distance to the cluster centres of the farthest $6 \mathrm{D}$ phase space member star. We note that the criterion to define the clusters' extensions (their radii) does not invalidate the discovery of the collision between IC 4665 and Collinder 350 which, at some level, is already indicated from Figure 1.

To analyze Collinder 350 and IC 4665 in more detail, we compiled lists of their member stars. For both the clusters, we adopted those stars which possess probability $P=1$ in Dias et al. (2021), which were recalculated using all the stars with membership probabilities $P=1$ in Cantat-Gaudin \& Anders (2020). To redetermine membership probabilities Dias et al. (2021) applied a variation of the classical maximum likelihood approach described in Dias et al. (2014) and Monteiro et al. (2020). Membership probabilities in CantatGaudin \& Anders (2020) were determined by employing the unsupervised UPMASK code (Krone-Martins \& Moitinho 2014). Fig. 2 depicts the colour-magnitude diagram built using Gaia DR2 $G, B P, R P$ photometry for these cluster members. For comparison purposes we corrected the magnitudes and colours by reddening using the mean cluster $E(B-V)$ values given by Dias et al. (2021), the heliocentric cluster distances mentioned above, and the $A_{\lambda} / A_{V}$ coefficiente given by Cardelli et al. (1989) and Wang \& Chen (2019). The fainter Main Sequence turnoff, the more pronounced curvature of the upper Main Sequence and the present of a 
red giant in Collinder 350 reveals its older age compared to IC 4665.

Bearing in mind that both clusters are physically separated by a distance smaller than the sum of their radii (see Fig. 1), their projected stellar structures should show evidence of such a closeness. We note that the stellar density profiles of tidally unperturbed clusters usually follow a nearly spherical King (1962) profile. Extended stellar envelopes/haloes, scattered stellar debris, tidal tails, etc, are instead found around clusters that experience tidal interactions (e.g., Pang et al. 2021).

Figure 3 shows the stellar density map of IC 4665 and Collinder 350. We build this map by using all cluster members in IC 4665 and Collinder 350, the scikit-learn software machine learning library (Pedregosa et al. 2011) and its kernel density estimator (KDE). We employed a grid of $500 \times 500$ boxes on the added cluster fields and allowed the bandwidth to vary from 0.1 up 0.5 in steps of 0.1 . Fig. 3 shows that the projected cluster circles, delineated by their tidal radii, intersect. We counted the number of IC 4665's members embraced within the Collinder 350's radius and found that $\sim 58$ percent of them are inside the later. IC 4665 , located to the north in this map, exhibits an elongated shape projected on the sky, while the outer region Collinder 350 seems to be azimuthally non uniform and presents some stellar debris. Both these features are commonly found in clusters experiencing tidal interaction (e.g., Dalessandro et al. 2015). Figure 4 shows that the projected stellar densities along the Declination axis reveal that stars of both clusters have crossed their respective tidal radii estimated by Piskunov et al. (2008). Indeed, when larger KDE bandwidths are used to build Fig. 3, so that large spatial scale structures can be highlighted, the line connecting both clusters (the gravitational bond line) along the Declination axis is apparent.

The analysis of stellar parallaxes also confirms the proximity of both clusters along the line-of-sight. To this end we used the Dias et al. (2021)'s parallaxes of every star with membership probabillity $P=1$, according to Dias et al. (2021) and Cantat-Gaudin \& Anders (2020). Both studies applied appropriate filters according to Gaia Collaboration et al. (2018b) to guarantee that only stars with good astrometric solutions are used. Stars resulted in cluster members from the likelihood approaches (Dias et al. 2021) and the $k$-means clustering technique (Cantat-Gaudin \& Anders 2020) have parallaxes and proper motions that belong to a concentrated group of values to the light of the uncertainties in proper motion, in parallax, and the correlation coefficients given in the Gaia DR2 catalogue. Fig. 5 shows the Gaia DR2 parallax distribution for cluster members in Collinder 350 and IC 4665, drawn with blue and red filled circles, respectively. We next determined the mean and dispersion of each cluster's parallax by employing a maximum likelihood approach, similar to the method detailed in Frank et al. (2015). The relevance lies in accounting for each individual star's measurement errors, which could artificially inflate the dispersion if ignored. We optimized the probability $\mathcal{L}$ that a given ensemble of stars with parallaxes $\varpi_{i}$ and parallax errors $\sigma_{i}$ are drawn from a population with mean parallax $\langle\varpi\rangle$ and intrinsic dispersion W (e.g., Walker et al.
2006; Koch et al. 2018), as follows:

$$
\begin{aligned}
\mathcal{L}=\prod_{i=1}^{N} & \left(2 \pi\left[\sigma_{i}^{2}+W^{2}\right]\right)^{-\frac{1}{2}} \\
& \times \quad \exp \left(-\frac{\left(\varpi_{i}-<\varpi>\right)^{2}}{\sigma_{i}^{2}+W^{2}}\right),
\end{aligned}
$$

where the errors on the mean and dispersion were computed from the respective covariance matrices. We note that this approach assumes that the error distribution is Gaussian, which is adopted here because of the limited number of stars (cf. Frank et al. 2015).

Table 1 shows the mean and dispersion for both clusters for different magnitude ranges (see Fig. 5). As can be seen, the parallax errors do not play a role; except possibly for the faintest stars, albeit the different number of stars in both clusters. We then defined a merger index as follows:

$$
\frac{(<\varpi>+W)_{\text {Collinder } 350-(<\varpi>-W}-(<C 4665}{W_{\text {Collinder } 350}+W_{I C} 4665},
$$

which measures in percentage the fraction of intersection between both clusters' volumes. The resulting merger indices show that both clusters are partially merged.

We took advantage of the proper motions, radial velocities and parallaxes of individual cluster members kindly provided by Dias et al. (2021) to compute their Galactic positions and space velocity components. We computed Galactic coordinates $(X, Y, Z)$ and space velocities $\left(V_{X}, V_{Y}, V_{Z}\right)$ employing the astropy ${ }^{1}$ package (Astropy Collaboration et al. 2013, 2018), which simply required the input of Right Ascension, Declination, parallaxes, proper motions and radial velocity of each star. We adopted the default values for the Galactocentric coordinate frame, namely : ICRS coordinates (RA, DEC) of the Galactic centre $=\left(266.4051^{\circ}\right.$, $\left.-28.936175^{\circ}\right)$; Galactocentric distance of the Sun $=8.122$ $\mathrm{kpc}$, height of the Sun above the Galactic midplane $=20.8$ pc; and solar motion relative to the Galactic centre $=(12.9$, $245.6,7.78) \mathrm{km} / \mathrm{s}$. The position of the Sun is assumed to be on the $X$ axis of the final, right-handed system. That is, the $X$ axis points from the position of the Sun projected to the Galactic midplane to the Galactic centre - roughly towards $(1, b)=\left(0^{\circ}, 0^{\circ}\right)$. The $Y$ axis points roughly towards Galactic longitude $l=90^{\circ}$, and the $Z$ axis points roughly towards the North Galactic Pole $\left(b=90^{\circ}\right)$.

Figure 6 illustrates a 3D view of the selected stars, which shows that not only IC 4665 but also Collinder 350 has an elongated shape. In order to build the figure we used the geometrical middle point between both clusters as the reference framework for positions and space motions, as this frame is also helpful to highlight their collision. Therefore, we used Galactic coordinates relative to that midpoint which is located at $(X, Y, Z)=(-7.82,0.17,0.12)$ $\mathrm{kpc}$, and have a velocity relative to the Galactic centre of $\left(V_{X}, V_{Y}, V_{Z}\right)=(5.54,231.80,5.41) \mathrm{km} / \mathrm{s}$. We performed this Galactic coordinate transformation in order to highlight the relative motions of both clusters. Indeed, the direction of the stellar space velocity vectors reveals that Collinder 350 and IC 4665 are traveling in opposite directions (in this adopted frame of reference). We refer the reader to the analysis of the disrupting open cluster Ruprecht 147, which shows similar

1 https://www.astropy.org 

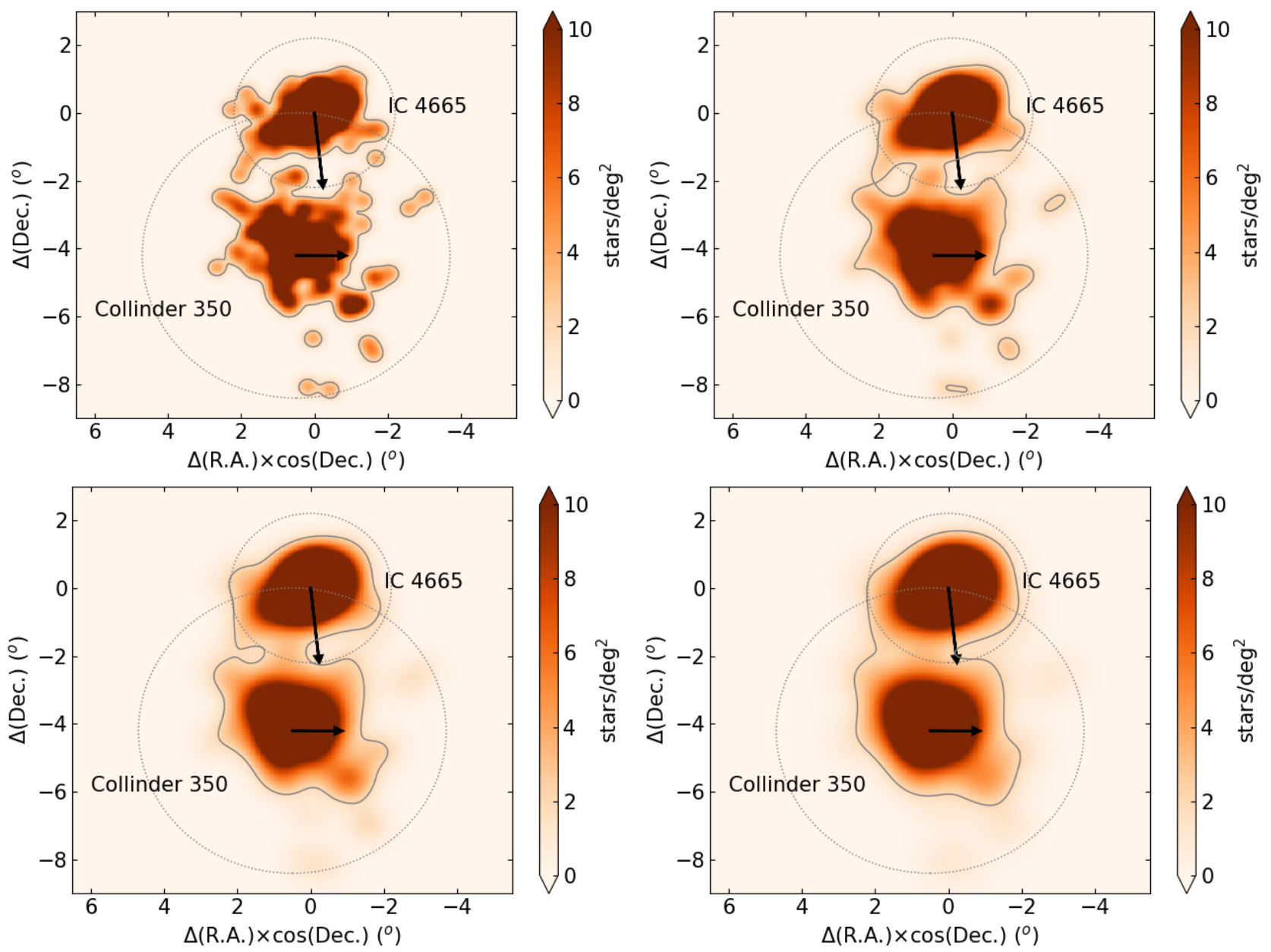

Figure 3. Stellar density maps of the partially merged cluster pair IC 4665 (northern cluster) and Collinder 350 . Dotted circles and black arrows represent the cluster adopted radii and proper motion vectors, respectively. A gray-coloured contour for a stellar density of 2 stars $/ \mathrm{deg}^{2}$ is also drawn. The maps were generated with KDE bandwidths of $0.2,0.3,0.4$, and 0.5 (from top to bottom, and from left to right), respectively.

Table 1. Parallax mean and dispersion values for Collinder 350 and IC 4665.

\begin{tabular}{cccccccc}
\hline & \multicolumn{3}{c}{ Collinder 350 } & \multicolumn{2}{c}{ IC 4665 } & \\
$M_{G}(\mathrm{mag})$ & $\varpi(\mathrm{mas})$ & $\mathrm{W}(\mathrm{mas})$ & $\mathrm{N}$ & $\varpi(\mathrm{mas})$ & $\mathrm{W}(\mathrm{mas})$ & $\mathrm{N}$ & merger index $(\%)$ \\
\hline$<-2.0$ & $2.72 \pm 0.03$ & $0.09 \pm 0.02$ & 14 & $2.90 \pm 0.01$ & $0.07 \pm 0.01$ & 14 & - \\
$-2.0-0.0$ & $2.71 \pm 0.01$ & $0.11 \pm 0.01$ & 30 & $2.87 \pm 0.03$ & $0.11 \pm 0.03$ & 18 & 27 \\
$0.0-2.0$ & $2.73 \pm 0.03$ & $0.12 \pm 0.02$ & 35 & $2.86 \pm 0.02$ & $0.08 \pm 0.01$ & 27 & 35 \\
$2.0-4.0$ & $2.67 \pm 0.01$ & $0.11 \pm 0.01$ & 33 & $2.85 \pm 0.03$ & $0.13 \pm 0.03$ & 25 & 27 \\
$>4.0$ & $2.74 \pm 0.07$ & $0.11 \pm 0.60$ & 8 & $2.80 \pm 0.05$ & $0.10 \pm 0.05$ & 55 & 68 \\
\hline
\end{tabular}

stellar structures (Yeh et al. 2019). We also constructed the right panel of Fig. 6 that confirms the collisional nature of the interaction between these two clusters; where blue/red arrows are computed with respect to the assumed centre of mass (middle point between both clusters). By using the centres of mass of each clusters and their respective stars, we built Fig 6 (bottom panels), which illustrate the internal motions of them. As can be seen, stars at opposite sides of the clusters move in opposite directions. Although the number of stars considered is small, these trends suggest that the clusters are in the process of disruption.

\section{THE CLUSTER ORBITS}

It is important to understand whether IC 4665 and Collinder 350 represent the merging of two objects in a binary cluster system (where the two clusters were originally formed together), or whether we are witnessing an actual collision between two independent star clusters. One way to probe this scenario is to integrate the orbits of these two clusters backwards in time and then examine the physical separation between the two clusters in the past, particularly at the birth time of the younger cluster IC 4665 (60 Myr ago). If this physical separation in the past turns out to 


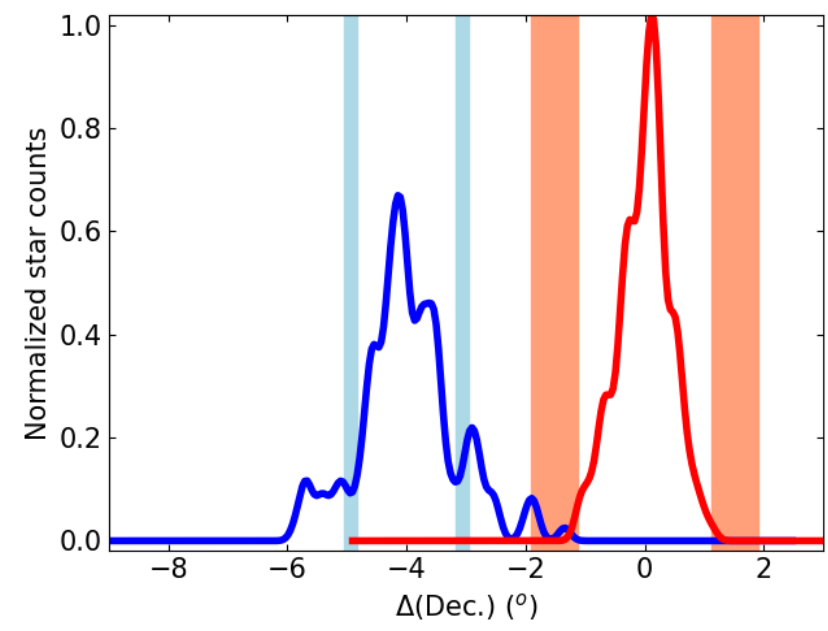

Figure 4. Projected stellar density map along the Declination axis. IC 4665 and Collinder 350 are drawn with red and blue lines respectively. The vertical shaded regions represent the tidal radii and their uncertainties as estimated by Piskunov et al. (2008). Stars of both clusters have escaped their respective tidal volumes.

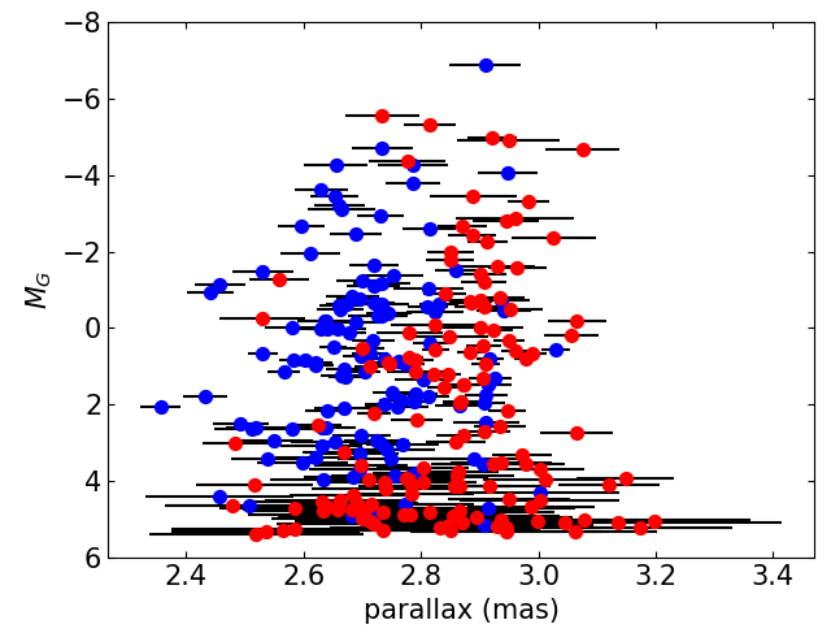

Figure 5. Relation between the absolute magnitudes and the parallaxes of stars drawn in Fig. 2. Blue and red filled cirles correspond to Collinder 350 and IC 4665, respectively. Individual error bars are also included. The data were kindly provided by W. Dias.

be large enough, then that would imply that IC 4665 and Collinder 350 have distinct physical origin, and thus represent independent star clusters. We note also that their ages differ by $\gtrsim 500 \mathrm{Myr}$.

To compute the orbits of these two open clusters (that are located in the Galactic disk), we follow a similar procedure described in Malhan et al. (2021) to integrate the orbits of globular clusters (that are otherwise located in the Galactic halo). Briefly, we use the measured heliocentric phase-space positions of the clusters and integrate them backward in time for $\sim 80 \mathrm{Myr}$. To integrate orbits, we try two different Galactic mass models that are amongst the best recommended in the literature - McMillan17 from McMillan (2017) and MWPotential2014 from Bovy (2015). Both the models are quite similar in construction: they both are axisymmetric models, each comprising a bulge, disk components and an NFW halo; except that McMillan17 overall is slightly heavier than MWPotential2014. The main reason for trying these two different potential models is to ensure that the final results are independent of the assumed Galactic mass model. Finally, in order to account for the measured uncertainties in phase-space associated with the clusters, we sample 1000 orbits for each cluster. The orbit analysis indicates that both the clusters have very disk-like orbits, with $Z$-component of angular momentum $L_{z} \sim-1830 \mathrm{~km} \mathrm{~s}^{-1} \mathrm{kpc}$ (implying prograde motion) and eccentricity $0-0.1$ (implying circular trajectories), although Collinder 350 has a slightly larger value of $L_{z}$ than IC 4665, due to Collinder 350's larger velocity.

Figure 5 compares the past orbits of IC 4665 and Collinder 350. It immediately reveals that the two clusters were physically separated in the past, but their orbital evolution has brought them closer to each other at the present day. We infer that the two clusters have only slightly different velocities, with Collinder 350 moving $\sim 5 \mathrm{~km} \mathrm{~s}^{-1}$ faster than IC 4665.

Figure 6 shows the relative (3D) physical separation between the clusters as a function of the lookback time. The two bold curves correspond to those orbits computed using the mean of the measured phase-space positions of the two clusters. The light shaded curves correspond to the orbits computed by sampling over the phase-space uncertainties. As can be easily discerned, this figure implies that IC 4665 and Collinder 350 were physically separated in the past, and that it is for the first time that they have come so close to each other at the present day. This implies that the two clusters have very different physical origin and were not born at the same location in our Galaxy. In other words, IC 4665 and Collinder 350 are not binary systems, but independent open clusters. This confirms our initial hypothesis that these two clusters represent a scenario of an actual collision between two independent star clusters.

It is also interesting to quantify the physical separation between IC 4665 and Collinder 350 at the birth time of the younger cluster IC 4665 . For this, we essentially take a slice of Figure 6 at $T=-60 \mathrm{Myr}$, and marginalise over the uncertainties to produce Figure 7 . With this we infer that $60 \mathrm{Myr}$ ago, IC 4665 and Collinder 350 were physically separated by a distance of $\gtrsim 500 \mathrm{pc}$ (specifically by $515_{-111}^{+239} \mathrm{pc}$ as per McMillan17 model and $551_{-112}^{+226} \mathrm{pc}$ as per MWPotential2014 model). As one can note, this relative distance between the two clusters is much larger than their combined physical radii.

\section{DISCUSSION AND CONCLUSION}

We have provided the first evidence of an on-going collision between two star clusters, namely IC 4665 and Collinder 350. This result is based on the analysis of the stellar density mapping of the two clusters, their ages, their present phasespace configuration and also their past orbital evolution in the Galaxy. Since these two clusters were formed at different times $(\Delta$ age $\lesssim 500 \mathrm{Myr})$ and at different locations in the galaxy (with a physical separation of $\gtrsim 500 \mathrm{pc}$ ), this implies that these two clusters do not represent merging of two objcets in a binary system, but instead, what we are witnessing 

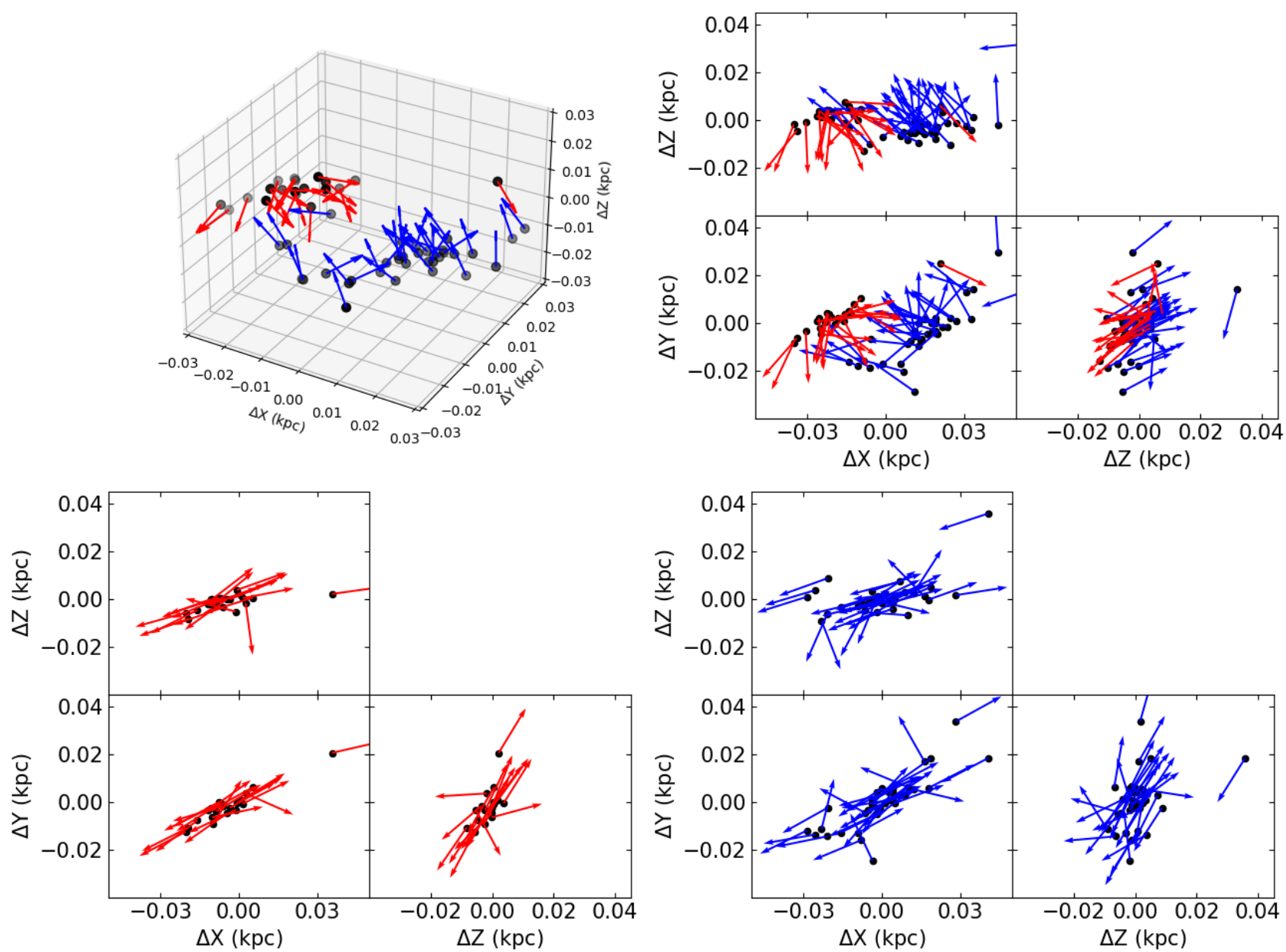

Figure 6. Left-top panel: 3D view of IC 4665 and Collinder 350 members (black dots) with their space velocity vectors relative to the motion of the centre of mass (assumed to be the geometrical middle point between both clusters), drawn with red and blue arrows, respectively. Right-top panel: projected space velocity vectors of IC 4665 and Collinder 350, with respect to the motion of their centre of mass. The Sun is placed at $\Delta X=7.82 \mathrm{kpc}, \Delta Y=-0.17 \mathrm{kpc}, \Delta Z=-0.12 \mathrm{kpc}$. Left-bottom panel: projected space velocity vectors of IC 4665 with respect to the motion of its centre. Right-bottom: same as for IC 4665, for Collinder 350.
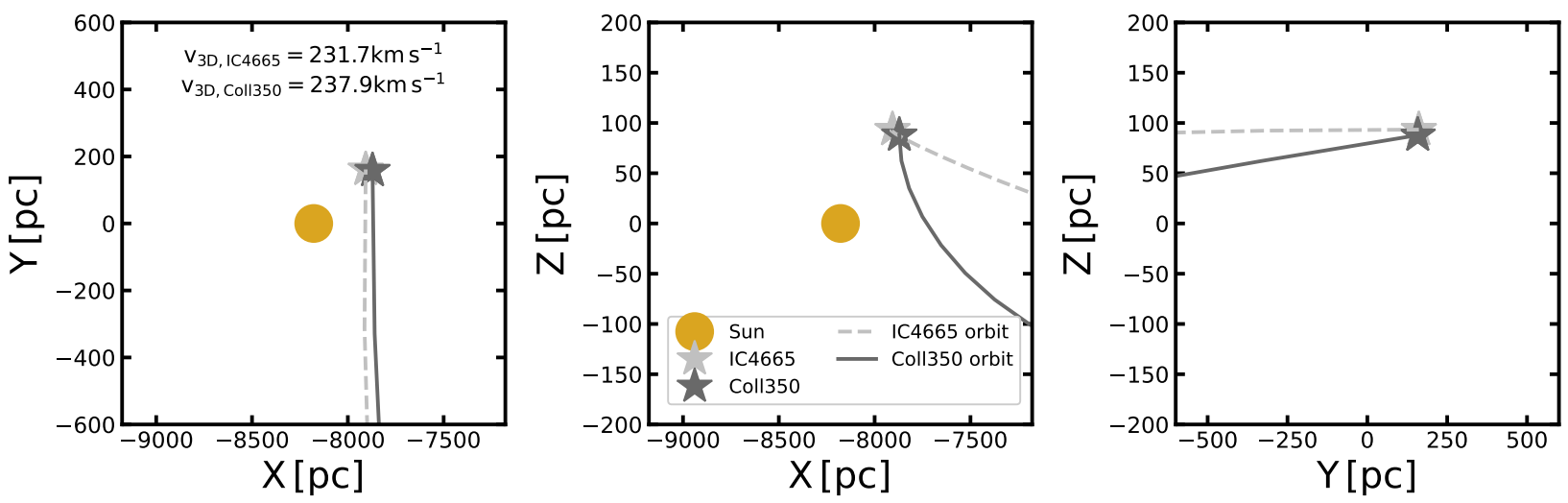

Figure 7. The figure is in the Cartesian coordinates, showing the present day positions and past orbits of IC 4665 and Collinder 350 . It clearly reveals that these two clusters were physically separated in the past, and it is for the first time that they have come so close to each other at the present day. The total linear Galactocentric velocities of the two clusters are provided in the plot (Collinder 350 is moving $\sim 5 \mathrm{~km} \mathrm{~s}^{-1}$ faster that IC 4665$)$. 


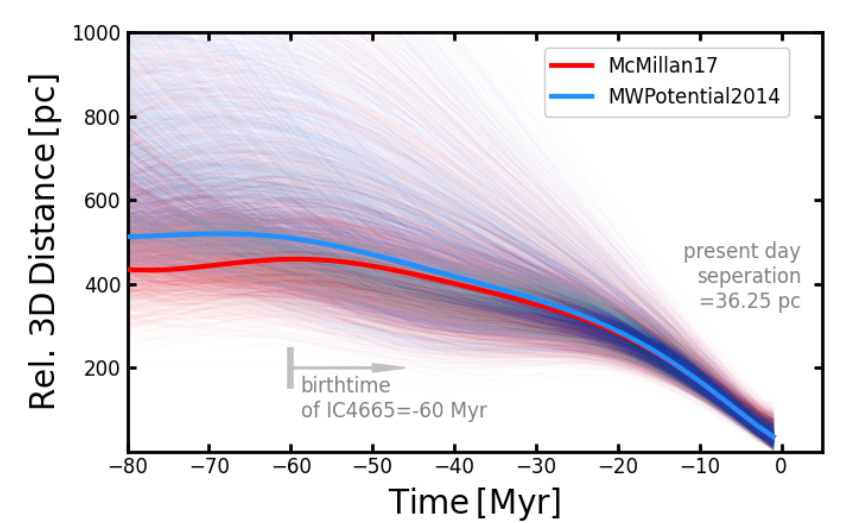

Figure 8. Time evolution of the relative 3D distance between IC 4665 and Collinder 350 ( $T=0$ represents the present day). We integrated orbits in two Galactic potential models (namely MWPotential2014 and McMillan17). The bold curves represent the orbits computed using mean phase-space positions of the clusters. The light shaded curves correspond to the orbits computed by sampling over the measured uncertainties in phase-space values.

is an actual collision between two independently formed star clusters.

In future work, it will be interesting to examine whether IC 4665 and Collinder 350 are coalescing into a single star cluster (that will essentially exhibit multiple stellar population with ages $=53 \mathrm{Myr}$ and $617 \mathrm{Myr}$ ), or whether the two clusters are simply passing-by each other (and undergoing only a tidal interaction) but will ultimately evolve as independent clusters. Future N-body simulations will be very useful to analyse this scenario in detail. In case the simulations find that the two clusters are coalescing, it will be important to learn about the final dynamical properties of the resulting system - does this merged system possesses a net rotation? does the density of this system differs from that of the initial colliding clusters? Moreover, if the simulations indeed favour the "coalescing" scenario, then that would further imply that observations of age/metallicity spread in star clusters do not necessarily always require multiple episodes of star formation, but that it can also occur as a result of collision between two star clusters hosting different stellar populations. This would effectively mean that the coalescence of multiple systems may be important in the history of at least some star clusters. Future analysis of IC 4665 and Collinder 350 will allow us to learn more about the detectable observational signatures of collision between independent star clusters.

The on-going collision between IC 4665 and Collinder 350 is not the prototypical phenomenon proposed by theories of the formation of star clusters, however, its discovery is both encouraging and interesting. Therefore, these two clusters provide a unique laboratory to explore various new aspects of formation and evolution theory of star clusters.

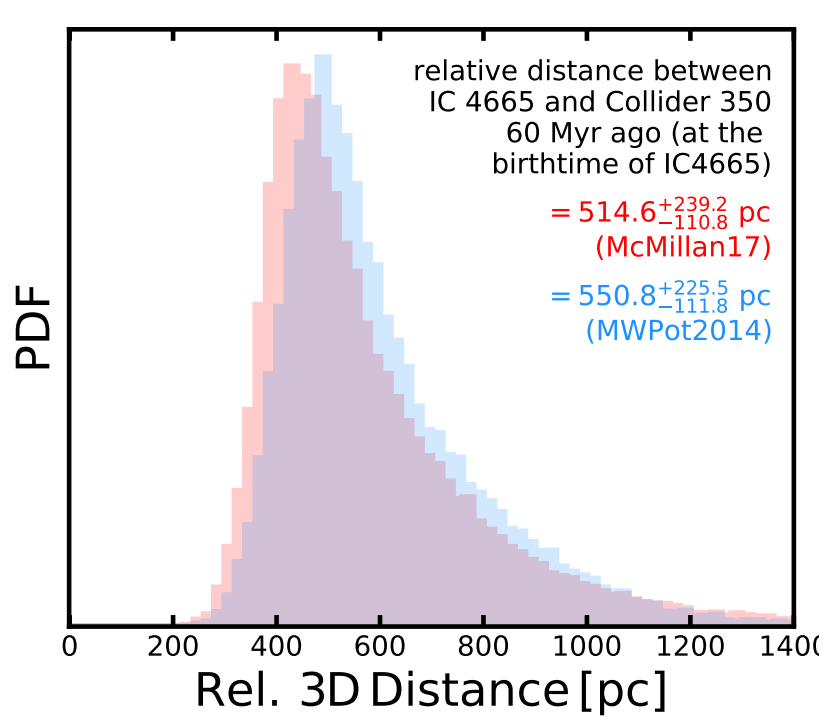

Figure 9. The probability distribution function (PDF) of the relative (3D) distance between IC 4665 and Collinder 350 in the past (60 Myr ago, at the birth time of IC 4665). This plot is essentially obtained by marginalising over the uncertainties in Figure 6 at $T=-60 \mathrm{Myr}$. Note that for both the trial Galactic potential models, we obtain a very similar PDF. For instance, the McMillan17 model indicates that $60 \mathrm{Myr}$ ago, the physical separation between the two clusters was $515_{-111}^{+239}$ pc. This suggests that IC 4665 and Collinder 350 originated at different physical location in the Galaxy, and they represent independent star clusters (and not a binary system).

\section{DATA AVAILABILITY}

Data used in this work are available upon request to the first author.

\section{ACKNOWLEDGEMENTS}

We warmly thank Wilton Dias for providing with the tables of membership probabilities and astrometric information for stars in the field of the studied open clusters, and his catalogue of astrophysical properties of 1743 open clusters. KM acknowledges support from the Alexander von Humboldt Foundation at Max-Planck-Institut für Astronomie, Heidelberg. KM is also grateful to the IAU's Gruber Foundation Fellowship Programme for their financial support. We thank the referee for the thorough reading of the manuscript and suggestions to improve it.

\section{REFERENCES}

Astropy Collaboration et al., 2013, A\&A, 558, A33

Astropy Collaboration et al., 2018, AJ, 156, 123

Bica E., Pavani D. B., Bonatto C. J., Lima E. F., 2019, AJ, 157, 12

Bovy J., 2015, ApJS, 216, 29

Calamida A., et al., 2007, ApJ, 670, 400

Cantat-Gaudin T., Anders F., 2020, A\&A, 633, A99

Cantat-Gaudin T., et al., 2019, A\&A, 626, A17

Cardelli J. A., Clayton G. C., Mathis J. S., 1989, ApJ, 345, 245 


\section{A.E. Piatti and K. Malhan}

Conrad C., et al., 2017, A\&A, 600, A106

Dalessandro E., Miocchi P., Carraro G., Jílková L., Moitinho A., 2015, MNRAS, 449, 1811

Dias W. S., Monteiro H., Caetano T. C., Lépine J. R. D., Assafin M., Oliveira A. F., 2014, A\&A, 564, A79

Dias W. S., Monteiro H., Moitinho A., Lépine J. R. D., Carraro G., Paunzen E., Alessi B., Villela L., 2021, MNRAS, 504, 356

Feigelson E. D., et al., 2011, ApJS, 194, 9

Frank M. J., Koch A., Feltzing S., Kacharov N., Wilkinson M. I., Irwin M., 2015, A\&A, 581, A72

Gaia Collaboration et al., 2016, A\&A, 595, A1

Gaia Collaboration et al., 2018a, A\&A, 616, A1

Gaia Collaboration et al., 2018b, A\&A, 616, A10

Kerr R. M. P., Rizzuto A. C., Kraus A. L., Offner S. S. R., 2021, ApJ, 917, 23

King I., 1962, AJ, 67, 471

Koch A., Hansen T. T., Kunder A., 2018, A\&A, 609, A13

Kounkel M., et al., 2018, AJ, 156, 84

Krone-Martins A., Moitinho A., 2014, A\&A, 561, A57

Lim D., Hong S., Lee Y.-W., 2017, ApJ, 844, 14

Malhan K., Yuan Z., Ibata R., Arentsen A., Bellazzini M., Martin N. F., 2021, arXiv e-prints, p. arXiv:2104.09523

McMillan P. J., 2017, MNRAS, 465, 76

Meingast S., Alves J., Rottensteiner A., 2021, A\&A, 645, A84

Monteiro H., Dias W. S., Moitinho A., Cantat-Gaudin T., Lépine J. R. D., Carraro G., Paunzen E., 2020, MNRAS, 499, 1874

Pang X., Li Y., Yu Z., Tang S.-Y., Dinnbier F., Kroupa P., Pasquato M., Kouwenhoven M. B. N., 2021, ApJ, 912, 162

Pedregosa F., et al., 2011, Journal of Machine Learning Research, 12,2825

Piskunov A. E., Schilbach E., Kharchenko N. V., Röser S., Scholz R. D., 2008, A\&A, 477, 165

Soubiran C., et al., 2018, A\&A, 619, A155

Steinmetz M., et al., 2006, AJ, 132, 1645

Townsley L. K., Broos P. S., Garmire G. P., Anderson G. E., Feigelson E. D., Naylor T., Povich M. S., 2018, ApJS, 235, 43

Walker M. G., Mateo M., Olszewski E. W., Bernstein R., Wang X., Woodroofe M., 2006, AJ, 131, 2114

Wang S., Chen X., 2019, ApJ, 877, 116

Yeh F. C., Carraro G., Montalto M., Seleznev A. F., 2019, AJ, 157,115

de La Fuente Marcos R., de La Fuente Marcos C., 2009, A\&A, 500, L13

de la Fuente Marcos R., de la Fuente Marcos C., 2010, ApJ, 719, 104

This paper has been typeset from a $\mathrm{T}_{\mathrm{E}} \mathrm{X} / \mathrm{LAT}_{\mathrm{E}} \mathrm{X}$ file prepared by the author. 